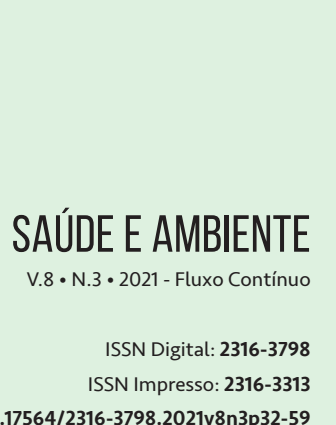

DOI: $10.17564 / 2316-3798.2021 v 8 n 3 p 32-59$

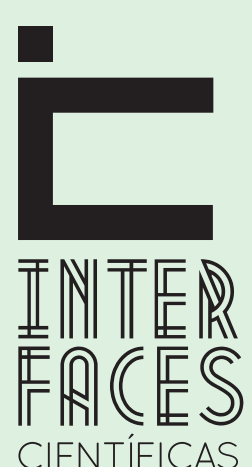

\section{HELMINTOS PARASITOS DE RÉPTEIS E ANFÍBIOS NO ESTADO DE SÃO PAULO, BRASIL}

HELMINTH PARASITES OF REPTILES AND AMPHIBIANS IN THE STATE OF SÃO PAULO, BRAZIL

HELMINTOS PARÁSITOS DE REPTILES Y ANFIBIOS EN EL ESTADO DE SÃO PAULO, BRASIL

Alessandra Aguirra Sani ${ }^{1}$ Geza Thaís Rangel e Souza²

Lívia Cristina dos Santos ${ }^{3}$

Tarsila Ferraz Frezza ${ }^{4}$

\section{RESUMO}

Os parasitos dizem muito sobre a biologia de seus hospedeiros, uma vez que revelam diferentes informações a respeito dos hábitos alimentares, ambientes onde vivem, rotas de migração do hospedeiro entre outras. Estudos relacionados à helmintologia com enfoque médico nos proporcionam um amplo conhecimento dos helmintos que infectam humanos, porém, entender as formas parasitárias em animais não humanos é também importante, pois trata-se de um meio de ampliar as informações sobre o parasitismo e suas interações. Devido à escassez de dados sobre a fauna helmintológica de anfíbios e répteis no estado de São Paulo, além da necessidade de se compilar dados a respeito do parasitismo por helmintos nestes animais, o presente trabalho teve por objetivo apresentar uma relação das espécies de helmintos associados a esses hospedeiros, com registro de ocorrência no estado de São Paulo. De acordo com a pesquisa realizada, os helmintos parasitos mais comuns para répteis e anfíbios no estado de São Paulo são os nematódeos de gênero Rhabdias, seguido de Physaloptera; foram também reportados trematódeos, cestódeos, acantocéfalos e monogêneas. Das 236 espécies de anfíbios existentes no Estado, apenas 27 (11,44\%) foram reportadas como hospedeiras para helmintos. Para os répteis, das 226 espécies encontradas no Estado, apenas 28 (12,39\%) foram reportadas como hospedeiras de helmintos. Esses dados evidenciam a necessidade e a importância de mais estudos sobre a helmintofauna de anfíbios e répteis.

\section{PALAVRAS-CHAVE}

Reptilia. Amphibia. Fauna Helmintológica. Parasita. Levantamento. 


\section{ABSTRACT}

Parasites reveal various information about the biology of their hosts, such as eating habits, environments in which they live and host migration routes. Studies in helminthology with a medical approach provide us with a broad knowledge of helminths that infect humans, however, understanding the parasitic forms in non-human animals is also important, as it is a means of expanding information about parasitism and ecological interactions. Due to the scarcity of data about helminthological fauna of amphibians and "reptiles", especially in the state of São Paulo-Brazil, and the need to compile data about helminth parasitism in these animals, the present study aimed to show a list of species of helminths associated with these vertebrate hosts that occur in the state of São Paulo. According to the present research, the most common parasitic helminths for "reptiles" and amphibians in the state of São Paulo are nematodes of the genus Rhabdias, followed by Physaloptera; however, trematodes, cestodes, acanthocephalus and monogeneans have also been reported. Regarding the 236 species of amphibians in São Paulo state, only 27 (11.44\%) were reported as hosts for helminths. Considering the "reptiles", 226 species are found in São Paulo state but only 28 (12.39\%) were reported as helminth hosts. These data show the need and the importance of further studies about amphibian and "reptile" helminth fauna.

\section{KEYWORDS}

Reptilia. Amphibia. Helminth Fauna. Parasite. Survey.

\section{RESUMEN}

Los parásitos dicen mucho sobre la biología de sus hospedadores, ya que revelan información diferente sobre los hábitos alimenticios, los entornos en los que viven, las rutas de migración de los hospedadores y otros. Los estudios relacionados con la helmintología con enfoque médico nos proporcionan un amplio conocimiento de los helmintos que infectan a los humanos, sin embargo, es importante comprender las formas parasitarias en animales no humanos, ya que es un medio para expandir la información sobre el parasitismo y sus interacciones ecológicas. Debido a la escasez de datos sobre la fauna helmintológica de anfibios y “reptiles”, especialmente en el estado de São Paulo-Brasil. Además de la necesidad de reunir datos sobre parasitismo de helmintos en estos animales, el presente estudio tuvo como objetivo presentar una lista de especies de helmintos asociados con estos hospedadores, con un registro de ocurrencia para el estado de São Paulo. Según la investigación realizada, los helmintos parásitos más comunes para "reptiles" y anfibios en el estado de São Paulo son los nematodos del género Rhabdias, seguidos de Physaloptera; sin embargo, se han reportado 
trematodos, cestodos, acanthocephalos y monogenéticos. De las 236 especies de anfibios presentes en el estado de São Paulo, solo 27 (11.44\%) fueron reportadas como hospedadores de helmintos. Estos datos nos muestran la necesidad y la importancia de realizar más estudios sobre la fauna de helmintos de anfibios y "reptiles".

\section{PALVABRAS-CLAVE}

Reptilia, Amphibia, Fauna Helmintológica, Parásitos, Levantamiento.

\section{INTRODUCÇÃO}

Sabe-se que os parasitos auxiliam na compreensão sobre a biologia de seus hospedeiros, uma vez que revelam diferentes informações a respeito dos hábitos alimentares, do ambiente onde vivem, das rotas de migração do hospedeiro, entre outras. Dentre os diferentes ramos da parasitologia, a helmintologia é aquela que se ocupa do estudo sobre os helmintos (AMATO; AMATO, 2010).

A helmintologia com enfoque médico é cada vez mais estudada, existindo um amplo conhecimento a respeito de helmintos que infectam e que produzem doenças em seres humanos. Todavia, uma vez que as formas parasitárias são diversas, é importante o entendimento dessas em animais não humanos, visando não apenas elucidar as formas de transmissão e prevenção de doenças para o ser humano (zoonoses), como também ampliar o arcabouço de informações sobre o parasitismo e as diferentes interações ecológicas existentes neste tipo de relação (SCHÜLLER, 2004).

Dentre os diferentes animais silvestres, o estudo da fauna helmintológica de répteis e anfíbios ainda é bastante escasso na literatura, embora o Brasil abrigue grande diversidade de répteis e anfíbios (COSTA; BÉRNILS, 2018; SEGALLA et al., 2019). De acordo com o American Museum of Natural History (AMNH, 2018), são descritas atualmente 7.926 espécies de anfíbios (Tabela 1). No país são reconhecidas 1.137 espécies de anfíbios, sendo 1.094 espécies de anuros, divididas em 20 famílias e 102 gêneros; 37 espécies e 12 gêneros de Gymnophiona, distribuídas em quatro famílias, e cinco espécies de Caudata, distribuídas em uma família e gênero (SEGALLA et al., 2019).

Até 2011, haviam sido registradas no estado de São Paulo as famílias de anfíbios: Caeciliidae (Gymnophiona) com seis espécies descritas; ordem Anura contendo as famílias Brachycephalidae (17 espécies), Bufonidae (oito espécies), Centrolenidae (duas espécies), Ceratophryidae (uma espécie), Craugastoridae (uma espécie), Cycloramphidae (23 espécies), Hemiphractidae (seis espécies), Hylidae (101 espécies), Hylodidae (19 espécies), Leiuperidae (18 espécies), Leptodactylidae (20 espécies), Microhylidae (12 espécies) e Strabomantidae (duas espécies) (ROSSA-FERES et al., 2011).

Em relação aos répteis, são reconhecidas 10.793 espécies no mundo (UETZ; HOSEK, 2018). No Brasil é relatada a ocorrência de 795 espécies de répteis, sendo 36 Testudines, seis Crocodylia e 753 Squamata (72 anfisbenas, 276 lagartos e 405 serpentes). Considerando as subespécies, existem seis 
Crocodylia, 37 Testudines e 799 Squamata no Brasil (75 anfisbenas, 282 lagartos e 442 serpentes), totalizando 842 espécies e subespécies de répteis no país (COSTA; BÉRNILS, 2018). No estado de São Paulo estão presentes 10 espécies da ordem Testudines, três espécies da ordem Crocodylia e 213 espécies de Squamata - 47 espécies de “lagartos", 10 espécies da subordem Amphisbaenia e 156 serpentes - totalizando 226 espécies de répteis (COSTA; BÉRNILS, 2018).

Devido à escassez de dados sobre a fauna helmintológica de anfíbios e répteis no Brasil e, especialmente, no estado de São Paulo, há uma necessidade de se compilar dados a respeito do parasitismo por helmintos nos animais existentes nesta região. Dessa forma, o presente trabalho buscou apresentar uma relação das espécies de helmintos associados a esses hospedeiros, com registro de ocorrência no estado de São Paulo.

\section{MÉTODO}

A relação de répteis e anfíbios com os helmintos endoparasitos a eles associados, no estado de São Paulo, foi obtida a partir de pesquisas bibliográficas nas seguintes bases de dados on-line: Google Acadêmico, Jstor, Pub Med, Scielo, Science Direct, Scopus e outras disponíveis no Portal de Periódicos da Coordenação de Aperfeiçoamento de Pessoal de Nível Superior (CAPES), em toda a literatura disponível nas respectivas bases, incluindo dissertações e teses. A pesquisa bibliográfica foi realizada até março de 2020.

Para a realização da pesquisa foram utilizados os seguintes termos: Acanthocephala, acantocéfalo(s), acanthocephalan(s), Digenea, digenean(s) digenético(s), Cestoda, cestode(s), cestódeo(s), cestóide(s), helminto(s), helminth(s), Nematoda, nematódeo(s), nematóide(s), nematode(s), Nemathelminthes, nemathelminth(s) nematelminto(s), parasito(s), parasita(s), parasite(s), parasitic, Platyhelminthes, platyhelminth(s) platelminto(s), São Paulo, Trematoda, trematódeo(s), trematode(s).

Cada um desses descritores, foram relacionados aos seguintes termos: Amphibia, amphibian(s), Amphisbaenia, anfíbio(s), Anura, Brachycephalidae, Bufonidae, Caeciliidae, Centrolenidae, Ceratophryidae, Craugastoridae, Crocodylia, Cycloramphidae, Gymnophiona, Hemiphractidae, Hylidae, Hylodidae, lagarto(s), Leiuperidae, Leptodactylidae, Microhylidae, Ophidia, répteis, réptil, reptile(s), Reptilia, Strabomantidae, Sauria, serpentes, Squamata, Testudines.

Foram consideradas todas as espécies de répteis e anfíbios, inclusive as exóticas e invasoras. As espécies de helmintos foram classificadas, conforme proposto por Tavares e colaboradores (2017). As nomeações taxonômicas foram utilizadas de acordo com os trabalhos originais (sem atualizações taxonômicas).

A proporção relativa entre a quantidade de espécies de répteis e anfíbios existentes no Estado de São Paulo foi calculada conforme proposto por Tavares e colaboradores (2017), tendo por base a quantidade de espécies destes vertebrados no referido Estado relatada por Rossa-Feres e colaboradores (2011) e por Costa e Bérnils (2018). 


\section{RESULTADOS E DISCUSSÃO}

De acordo com a pesquisa bibliográfica realizada, foram identificados 28 gêneros e 34 espécies de helmintos em anfíbios. Dentre esses, foram reportados 13 gêneros de Nematoda; um gênero de Ancanthocephala, Cestoda e Monogenea; nove gêneros de Trematoda. Somente os anfíbios da ordem Anura (26 espécies identificadas) foram relacionados, atuando como hospedeiros para as espécies de helmintos no Estado de São Paulo.

Dentre os nematódeos parasitos de anuros, o gênero Rhabdias Stiles \& Hassall, 1905 foi o mais comum, parasitando 16 espécies. Em seguida, nematódeos do gênero Physaloptera Rudolphi, 1819 foram encontrados, parasitando 15 espécies de anuros. 0 gênero Rhabdias representou $27 \%$ do total de nematódeos encontrados, parasitando Anura (de um total de 64 nematódeos reportados), enquanto que Physaloptera representou 23\% (Figura 1A). A espécie de Anura com maior número de helmintos foi Boana raniceps, (COPE, 1862) com 10 espécies identificadas de helmintos.

Em relação aos répteis, foram levantados 57 gêneros e identificadas 51 espécies de helmintos; e 28 espécies de répteis foram reportadas, atuando como hospedeiros para helmintos no estado de São Paulo. Os hospedeiros foram duas espécies da Ordem Crocodylia, 12 espécies de Serpentes (Squamata), nove espécies de "Sauria" (Squamata) e cinco espécies de Testudines. Foram identificados e reportados 23 gêneros de Nematoda, parasitando répteis no estado de São Paulo; um gênero de Ancanthocephala; três gêneros de Cestoda; 24 gêneros de Trematoda e um de Monogenea.

Apesar de ter sido reportada maior quantidade de gêneros de trematódeos, parasitando répteis, os nematódeos foram os helmintos mais comuns, tendo sido encontrado em todas as famílias de répteis listadas. Dentre os gêneros de nematódeos, Rhabdias e Physaloptera foram os mais comuns (representando 15\%, cada, dos gêneros de Nematoda) (Figura 1B). Dentre as espécies de répteis, Chelonia mydas (LINNAEUS, 1758) (Testudines) foi a que apresentou maior número de helmintos (12 espécies). 
Figura 1 - Espécies de Nematódeos parasitos e sua porcentagem de ocorrência no estado de São Paulo. A. Anura. B. répteis

A
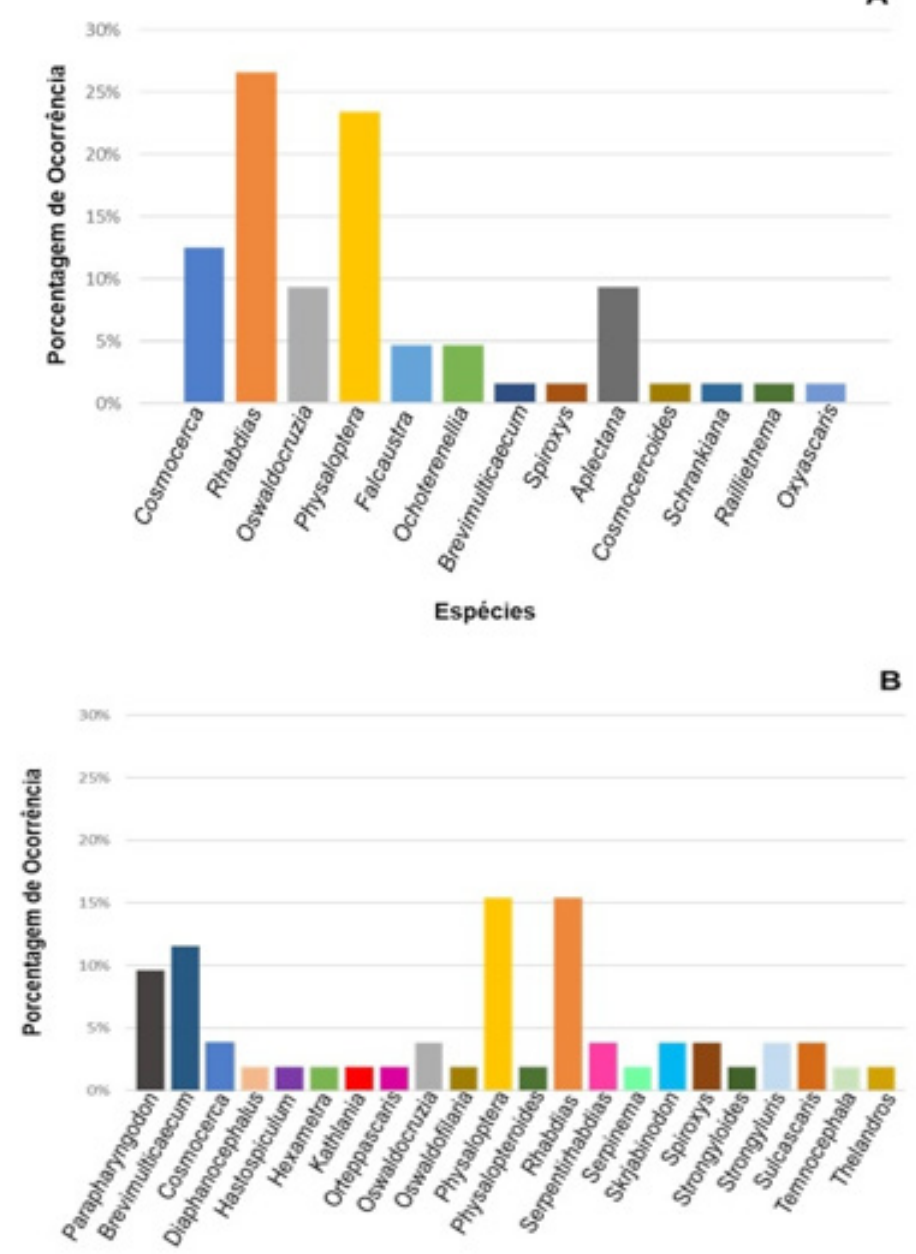

Fonte: Dados da pesquisa.

A maioria dos helmintos levantados nesta pesquisa pertencem ao filo Nematoda, tendo sido reportado 78 vezes como parasitos de anfíbios e 58 vezes, parasitando répteis; seguido pelo filo Platyhelminthes, classe Trematoda (22 vezes reportados como parasitos de anfíbios e 30 parasitando répteis); filo Acanthocephala (15 parasitando anfíbios e sete répteis); filo Platyhelminthes, classe Cestoda (cinco parasitando anfíbios e seis répteis) e filo Platyhelminthes, classe Monogenea (um parasitando anfíbios e dois répteis) (Figura 2). 
Figura 2 - Nematoda, Trematoda, Cestoda, Acanthocephala e Monogenea: distribuição dos helmintos nos hospedeiros répteis e Anura no estado de São Paulo

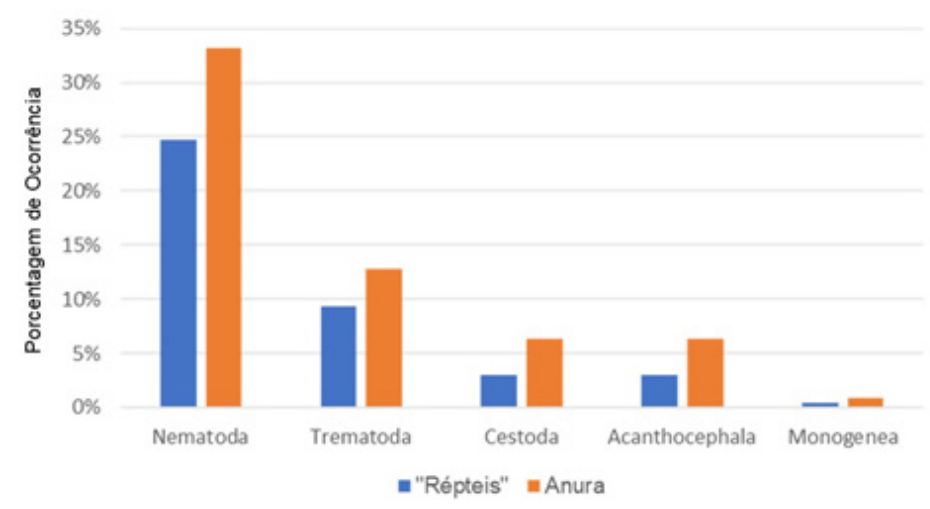

Fonte: Dados da pesquisa.

Das 236 espécies de anfíbios existentes no estado de São Paulo, apenas 27, ou seja 11,44\%, foram reportadas como hospedeiras para diferentes helmintos. Em relação aos répteis, das 226 espécies encontradas no Estado, apenas 28 (12,39\%) foram reportadas como hospedeiras de helmintos.

Os helmintos parasitas para as famílias de anuros e de répteis no estado de São Paulo estão listados a seguir:

\section{AMPHIBIA}

Anura

Brachycephalidae

Ischnocnema bolbodactyla (LUTZ, 1925)

Nematoda - larva sem identificação

Ischnocnema parva (GIRARD, 1853)

Cestoda (Nematotaenidae) - Cylindrotaenia americana Jewel, 1916

Nematoda (Cosmocercidae) - larva sem identificação

Ischnocnema guentheri(STEINDACHNER, 1864)

Acanthocephala (Echinorhynchidae) - Acanthocephalus saopaulensis Smales, 2007

Cestoda (Nematotaenidae) - Cylindrotaenia americana Jewel, 1916 
Nematoda (Cosmocercidae) - Cosmocerca parva Travassos, 1925

Nematoda (Rhabdiasidae) - Rhabdias sp. Stiles \& Hassall, 1905

\section{Bufonidae}

Dendrophryniscus brevipollicatus Jiménez de la Espada, 1870

Nematoda (Cosmocercidae) - Sem identificação

Rhinella ornata (SPIX, 1824)

Acanthocephala - cistos sem identificação

Nematoda (Molineoidea) - Oswaldocruzia sp. Travassos, 1917

Nematoda (Physalopteridae) - Physaloptera sp. Rudolphi, 1819

Nematoda (Rhabdiasidae) - Rhabdias sp. Stiles \& Hassall, 1905

\section{Rhinella icterica (SPIX, 1824)}

Acanthocephala (Echinorhynchidae) - Acanthocephalus saopaulensis Smales, 2007

Nematoda (Kathlaniidae) - Falcaustra mascula (RUDOLPHI, 1819)

Nematoda (Molineoidea) - Oswaldocruzia subauricularis (RUDOLPHI, 1819)

Nematoda (Physalopteridae) Physaloptera sp. Rudolphi, 1819

Nematoda (Rhabdiasidae) - Rhabdias sp. Stiles \& Hassall, 1905, Rhabdias fuelleborni (TRA VASSOS, 1926)

Trematoda (Mesocoeliidae) - Mesocoelium monas (Rudolphi, 1819)

\section{Craugastoridae}

Haddadus binotatus (SPIX, 1824)

Acanthocephala (Echinorhynchidae) - Acanthocephalus saopaulensis Smales, 2007

Nematoda (Cosmocercidae) - Cosmocerca parva Travassos, 1925, Cosmocerca brasiliensis Travassos, 1925

Nematoda (Molineoidea) - Oswaldocruzia sp. Travassos, 1917

Nematoda (Onchoceridae) - Ochoterenella sp. Caballero, 1944

Nematoda (Physalopteridae) - Physaloptera sp. Rudolphi, 1819 
Nematoda (Rhabdiasidae) - Rhabdias sp. Stiles \& Hassall, 1905

Hemiphractidae

Fritziana fissilis (MIRANDA-RIBEIRO, 1920)

Acanthocephala - Sem identificação

Nematoda (Physalopteridae) - Physaloptera sp. Rudolphi, 1819

Hylidae

Dendropsophus nanus (BOULENGER, 1889)

Acanthocephala - cistos sem identificação

Cestoda (Nematotaenidae) - Cylindrotaenia americana Jewel, 1916

Nematoda (Cosmocercidae) - Cosmocerca podicipinus Baker \& Vaucher, 1984

Nematoda (Heterocheilidae) - Brevimulticaecum sp. Mozgovoi, 1951 Nematoda (Spiruroidea) Spiroxys sp. Schneider, 1866

Trematoda (Clinostomidae) - Clinostomum sp. Leidy, 1856

Trematoda (Diplostomidae) - Lophosicyadiplostomum sp. Dubois, 1936

Trematoda (Paramphistomidae) - Catadiscus propinquus Freitas \& Dobin Jr., 1956

Boana albomarginata (SPIX, 1824)

Nematoda (Physalopteridae) - Physaloptera sp. Rudolphi, 1819

Nematoda (Rhabdiasidae) - Rhabdias sp. Stiles \& Hassall, 1905

Boana raniceps (COPE, 1862)

Acanthocephala (Centrorhynchidae) - sem identificação

Nematoda (Cosmocercidae) - Aplectana membranosa Schneider, 1866

Nematoda (Heterocheilidae) - Brevimulticaecum sp. Mozgovoi, 1951 Nematoda (Onchoceridae)

- Ochoterenellasp. Caballero, 1944

Nematoda (Physalopteridae) - Physaloptera sp. Rudolphi, 1819

Nematoda (Rhabdiasidae) - Rhabdias sp. Stiles \& Hassall, 1905

Trematoda (Diplostomidae) - Lophosicyadiplostomum sp. Dubois, 1936

Trematoda (Gorgoderidae) - Gorgoderina diaster Lutz, 1926 
Trematoda (Macroderoididae) - Choledocystus vitellinophilum (DOBBIN, 1958)

Trematoda (Paramphistomidae) - Catadiscus propinquus Freitas \& Dobin Jr., 1956

Boana albopunctata (SPIX, 1824)

Nematoda (Cosmocercidae) - Aplectana sp. Railliet \& Henri, 1916, Cosmocerca parva Travassos, 1925, Cosmocerca sp. Diesing, 1816, Cosmocercoides sp. Wilkie, 1930

Nematoda (Molineoidea) - Oswaldocruzia subauricularis (Rudolphi, 1819)

Nematoda (Physalopteridae) - Physaloptera sp. Rudolphi, 1819

Nematoda (Rhabdiasidae) - Rhabdias sp. Stiles \& Hassall, 1905

Trematoda (Haematoloechiidae) - Haematoloechus fuelleborni (TRAVASSOS; DARRIBA, 1930)

Boana faber (WIED-NEUWIED, 1821)

Nematoda (Atractidae) - Schrankianasp. Strand, 1942

Nematoda (Cosmocercidae) - Aplectana sp. Railliet \& Henri, 1916, Cosmocerca parva Travassos, 1925, Raillietnema simples Travassos, 1925

Nematoda (Kathlaniidae) - Falcaustra mascula (Rudolphi, 1819)

Nematoda (Molineoidea) - Oswaldocruzia subauricularis (RUDOLPHI, 1819)

Nematoda (Onchoceridae) - Ochoterenella sp. Caballero, 1944

Nematoda (Physalopteridae) - Physaloptera sp. Rudolphi, 1819

Nematoda (Rhabdiasidae) - Rhabdias sp. Stiles \& Hassall, 1905

Boana prasina (BURMEISTER, 1856)

Cestoda (Nematotaenidae) - Cylindrotaenia americana (JEWEL, 1916)

Nematoda (Physalopteridae) - Physaloptera sp.Rudolphi, 1819

Nematoda (Rhabdiasidae) - Rhabdias füelleborni Travassos, 1926

Boana polytaenia (COPE, 1870)

Nematoda (Rhabdiasidae) - Rhabdias sp. Stiles \& Hassall, 1905

Scinax hayii (BARBOUR, 1909) 
Nematoda (Physalopteridae) - Physaloptera sp. Rudolphi, 1819

Nematoda (Rhabdiasidae) - Rhabdias sp. Stiles \& Hassall, 1905

Hylodidae

Hylodes asper (MÜLLER, 1924)

Nematoda (Cosmocercidae) - sem identificação

Hylodes phyllodes Heyer \& Cocroft, 1986

Cestoda (Nematotaenidae) - Cylindrotaenia americana Jewel, 1916

Nematoda (Physalopteridae) - Physaloptera sp. Rudolphi, 1819

Leptodactylidae

Leptodactylus latrans (STEFFEN, 1815)

Acanthocephala - sem identificação

Nematoda - (Cosmocercidae) - Oxyascaris oxyascaris Travassos, 1920

Nematoda (Kathlaniidae) - Falcaustra mascula (RUDOPLHI, 1819)

Nematoda (Physalopteridae) - Physaloptera sp. Rudolphi, 1819

Adenomera marmorata Steindachner, 1867

Acanthocephala - sem identificação

Nematoda (Cosmocercidae) - Aplectana membranosa (Schneider, 1866)

Nematoda (Physalopteridae) - Physaloptera sp. Rudolphi, 1819

Nematoda (Rhabdiasidae) - Rhabdiassp. Stiles \& Hassall, 1905

Leptodactylus sp. Fitzinger, 1826

Nematoda (Cosmocercidae) - sem identificação

Leptodactylus chaquensis Cei, 1950

Acanthocephala (Centrorhynchidae) - sem identificação 
Nematoda (Heterocheilidae) - Brevimulticaecum sp. (MOZGOVOI, 1951)

Nematoda (Rhabdiasidae) - Rhabdias sp. Stiles \& Hassall, 1905

Trematoda (Diplostomidae) - Lophosicyadiplostomum sp. Dubois, 1936

Trematoda (Glypthelminthidae) - Rauschiella palmipedis (Lutz, 1928)

Trematoda (Paramphistomidae) - Catadiscus propinquus Freitas \& Dobin Jr., 1956

\section{Leptodactylus podicipinus (COPE,1862)}

Acanthocephala (Centrorhynchidae) - sem identificação

Nematoda (Cosmocercidae) - Aplectana membranosa (Schneider, 1866), Cosmocerca podicipinus Baker \& Vaucher, 1984

Nematoda (Heterocheilidae) - Brevimulticaecum (MOZGOVOI, 1951) Nematoda (Rhabdiasidae) Rhabdias sp. Stiles \& Hassall, 1905

Trematoda (Diplostomidae) - Heterodiplostomum sp. Dubois, 1936, Lophosicyadiplostomum sp. Dubois, 1936

Trematoda (Glypthelminthidae) - Rauschiella sp. Babero, 1951

Trematoda (Paramphistomidae) - Catadiscus sp. Cohn, 1904, Catadiscus marinholuitzi Freitas \& Lent, 1939

\section{Physalaemus cuvieri Fitzinger, 1826}

Acanthocephala (Echinorhynchidae) - Acanthocephalus saopaulensis Smales, 2007

Monogenea (Polystomatidae) - Polystoma cuvieri Vaucher, 1990

Nematoda (Cosmocercidae) - Aplectana sp. Railliet \& Henri, 1916

Nematoda (Molineoidea) - Oswaldocruzia subauricularis (RUDOLPHI, 1819)

Nematoda (Physalopteridae) - Physaloptera sp. Rudolphi, 1819

Nematoda (Rhabdiasidae) - Rhabdias sp. Stiles \& Hassall, 1905

Microhylidae

Chiasmocleis carvalhoi(NELSON, 1975)

Acanthocephala - sem identificação

Nematoda (Physalopteridae) - Physaloptera sp. Rudolphi, 1819 
Nematoda (Rhabdiasidae) - Rhabdias sp. Stiles \& Hassall, 1905

Myersiella microps (DUMÉRIL; BIBRON, 1841)

Acanthocephala - sem identificação

Ranidae

Lithobates catesbeianus (SHAW, 1802)

Nematoda (Cylindrocorporidae) - Longibucca catesbeianae De Souza Junior, Artigas \& Martins, 1993

\section{REPTILIA}

Crocodylia

Alligatoridae

Caiman yacare (DAUDIN, 1802)

Nematoda (Ascaridoidea) - Ortleppascaris alata (BAYLIS, 1947)

Paleosuchus palpebrosus (CUVIER, 1807)

Nematoda (Heterocheilidae) - Brevimulticaecum pintoi Sprent, 1979

\section{Serpentes}

Boidae

Eunectes murinus (LINNAEUS, 1758)

Cestoda (Proteocephalidae) - Crepidobothrium gerrardii (BAIRD, 1860)

Nematoda (Diplotriaenidae) - Hastospiculum digiticaudatum Teixeira \& Freitas, 1955

Colubridae

Chironius flavolineatus Jan, 1863

Nematoda (Rhabdiasidae) - Serpentirhabdias sp. Kach, Kuzmin \& Snyder, 2014

Chironius quadricarinatus Boie, 1827 
Acanthocephala - cistos sem identificação

Nematoda (Heterocheilidae) - Brevimulticaecum sp. Mozgovoi, 1951 Trematoda - Digenea (Dicro coelidae) - Infidum infidum Faria, 1910

Erythrolamprus poecilogyrus (WIED-NEUWIED, 1825)

Acanthocephala - cistos sem identificação

Trematoda - Digenea (Dicrocoelidae) - Infidum similis Travassos, 1916

Helicops infrataeniatus Jan, 1865

Nematoda (Heterocheilidae) - Brevimulticaecum sp. Mozgovoi, 1951

Trematoda (Dicrocoelidae) - Infidum similis Travassos, 1916

Pseudoboa nigra (DUMÉRIL; BIBRON; DUMÉRIL, 1854)

Acanthocephala - cistos sem identificação

Nematoda - cistos sem identificação

Nematoda (Heterocheilidae) - Brevimulticaecum sp. Mozgovoi, 1951 Nematoda (Physalopteri dae) - Physaloptera sp. Rudolphi, 1819

Nematoda (Spiruroidea) - Spiroxys figueireidoi Freitas \& Dobbin Jr., 1962

Dipsas mikanii(SCHLEGEL, 1837)

Nematoda (Rhabdiasidae) - Rhabdias sp. Stiles \& Hassall, 1905

Thamnodynastes hypoconia (COPE, 1860)

Acanthocephala - cistos sem identificação

Trematoda (Dicrocoelidae) - Infidum infidum Faria, 1910

Trematoda - Sem identificação

Spilotes pullatus (LINNAEUS, 1758)

Nematoda (Rhabdiasidae) - Rhabdias filicaudalis Barrella; Santos; Silva, 2010 
Elapidae

Micrurus frontalis Duméril, Bibron \& Duméril, 1854

Trematoda (Plagiorchiidae) - Haplometroides odhneri Ruiz \& Perez, 1959

Typhlopidae

Amerotyphlops brongersmianus (VANZOLINI, 1972)

Nematoda (Rhabdiasidae) - Rhabdias sp. Stiles \& Hassall, 1905

Viperidae

Bothrops moojeni (HOGE, 1966)

Acanthocephala - cistos sem identificação

Cestoda (Proteocephalidae) - Crepidobothrium gerrardii (Baird, 1860), Ophiotaenia sp. La Rue, 1911

Nematoda (Cosmocercidae) - Cosmocerca podicipinus Baker \& Vaucher, 1984

Nematoda (Heterocheilidae) - Brevimulticaecum sp. Mozgovoi, 1951 Nematoda (Physalopteridae)

- Physaloptera sp. Rudolphi, 1819

Nematoda (Rhabdiasidae) Rhabdias sp. Stiles \& Hassall, 1905

Nematoda (Strongyloididae) - Strongyloides ophidae Pereira, 1929

Trematoda (Dicrocoelidae) - Infidum infidum Faria, 1910

Trematoda (Plagiorchiidae) - Travtrema stenocotyle (COHN, 1902), Ophisthogonimus sp. (LÜEHE, 1990)

Trematoda (Sticholecithidae) - Sticholecitha serpentis Prudhoe, 1949

Crotalus durissus terrificus (LAURENTI, 1768)

Nematoda (Rhabdiasidae) - Rhabdias sp. Stiles \& Hassall, 1905, Serpentirhabdias sp. Tkach, Kuzmin \& Snyder, 2014

Anguimorpha

Anguidae

Ophiodes striatus (SPIX, 1824) 
Nematoda (Physalopteridae) - Physaloptera sp. Rudolphi, 1819

Nematoda (Rhabdiasidae) - Rhabdias sp. Stiles \& Hassall, 1905

Gekkota

Gekkonidae

Hemidactylus mabouia (MOREAU DE JONNÉS, 1818)

Acantocephala (cistos) - Sem identificação

Nematoda (Acuariidae) - Sem identificação.

Nematoda (Pharyngodonidae) - Parapharyngodon largitorAlho \& Rodrigues, 1963, Parapharyn godon sceleratus (Travassos, 1923), Parapharyngodon sp. (CHATTERJI, 1933)

Nematoda (Physalopteridae) - Physaloptera sp. Rudolphi, 1819

Lacertoidea

Gymnophthalmidae

Colobosaura modesta (REINHARDT; LÜTKEN, 1862)

Cestoda (Linstowiidae) - Oochoristica sp. Luhe, 1898

Teiidae

Ameiva ameiva (LINNAEUS, 1758)

Cestoda (Linstowiidae) - Oochoristica sp. (LUHE, 1898)

Nematoda (Pharyngodonidae) - Parapharyngodon largitorAlho \& Rodrigues, 1963

Salvator merianae Duméril \& Bilbron, 1839, Salvator rufescens (GÜNTHER, 1871), Salvator tegui xin (LINNAEUS, 1758)

Nematoda (Diaphanocephalidae) - Diaphanocephalus galeatus (RUDOLPHI, 1818)

Iguania

Leiosauridae

Enyalius iheringii Boulenger, 1885

Nematoda (Heterakidae) - Strongyluris oscari Travassos, 1923

Nematoda (Molineoidea) - Oswaldocruzia fredi Durette-Desset; Anjos et Vrcibradic, 2006 
Nematoda (Rhabdiasidae)- Rhabdias sp. Stiles \& Hassall, 1905

\section{Enyalius perditus Jackson, 1978}

Acantocephala (Echinorhynchidae) - Acanthocephalus sp. Kolreter, 1771

Nematoda (Cosmocercidae) - Cosmocerca sp. Diesing, 1861

Nematoda (Heterakidae) - Strongyluris oscari Travassos, 1923

Nematoda (Molineidae) - Oswaldocruzia burseyi Durette-Desset, Anjos et Vrcibradic, 2006

Nematoda (Rhabdiasidae) - Rhabdias sp. Stiles \& Hassall, 1905

Tropiduridae

Tropidurus oreadicus Rodrigues, 1987

Nematoda (Pharyngodonidae) - Parapharyngodon largitorAlho \& Rodrigues, 1963

Nematoda (Physalopteridae) - Physaloptera sp. Rudolphi, 1819, Physalopteroides sp. Wu \& Liu, 1940

\section{Scincoidea}

Scincidae

Aspronema dorsivittatum (COPE, 1862)

Cestoda (Linstowiidae) - Oochoristica sp. (LUHE, 1898)

Nematoda (Physalopteridae) - Physaloptera sp. Rudolphi, 1819

Trematoda - Sem identificação

Aspronema dorsivittata (COPE, 1862), Mabuya frenata (COPE, 1862)

Nematoda (Ascarididae) - Hexametra boddaertii (Baird, 1860)

Nematoda (Onchocercidae) - Oswaldofilaria sp. (PIRATUBA; LENT; FREITAS, 1941)

Nematoda (Pharyngodonidae) - Skrjabinodon sp. Inglis, 1968

Nematoda (Pharyngodonidae) - Skrjabinodon spinosulus Vicente; Vrcibradic; Rocha; Pinto, 2002

Nematoda (Pharyngodonidae) - Thelandros sceleratus Walton, 1941

Nematoda (Physalopteridae) - Physaloptera retusa (RUDOLPHI, 1819) 
Testudines

Chelidae

Hydromedusa tectifera Cope, 1869

Nematoda (Temnocephalidae) - Temnocephala brevicornis Monticelli, 1889

Phrynops geoffroanus (SCHWEIGGER, 1812)

Monogenea (Polystomatidae) - Polystomoides brasiliensi Vieira; Novelli; Souza; de Souza-Lima, 2008, Polystomoides sp. Ward, 1917

Nematoda - Sem identificação

Nematoda (Camallanidae) - Serpinema monospiculatus Freitas \& Dobbin Jr., 1962

Nematoda (Heterocheilidae) - Brevimulticaecum sp. Mozgovoi, 1951 Nematoda (Physalopteridae)

- Physaloptera sp. Rudolphi, 1819

Nematoda (Spiruroidea) - Spiroxys figuereidoi Freitas \& Dobbin Jr., 1962

Trematoda (Paramphistomidae) - Nematophila grandis Diesing, 1839

Trematoda (Proterodiplostomidae) - Cheloniodiplostomum testudinis (DUBOIS, 1936)

Trematoda (Telorchiidae) - Telorchis birabeni Mañé-Garzón \& Gil, 1961

\section{Cheloniidae}

Caretta caretta (LINNAEUS, 1758)

Nematoda (Anisakidae) - Sulcascaris sulcata (RUDOLPHI, 1819)

Nematoda (Kathlanidae) - Kathlania leptura (RUDOLPHI, 1819)

Trematoda (Calycodidae) - Calycodes anthos Braun, 1899

Trematoda (Pronocephalidae) - Pyelosomum renicapite (LEIDY, 1856)

Trematoda (Telorchiidae) - Orchidasma amphiorchis (BRAUN, 1889)

\section{Chelonia mydas (LINNAEUS, 1758)}

Trematoda (Angiodictyidae) - Deuterobaris proteus (BRANDES, 1891), Microscaphidium reticula re(VAN BENEDEN, 1859), Neoctangium travassosi Ruiz, 1943, Polyangium linguatula (LOOSS, 1899)

Trematoda (Pronocephalidae) - Cricocephalus albus (KUHL; HASSEL, 1822), Cricocephalus megastomum (LOOSS, 1902), Pleurogonius longiusculus Looss, 1901, Pronocephalus obliquus Looss, 1901, Metacetabulum invaginatum Freitas \& Lent, 1938 
Trematoda (Spirorchiidae) - Learedius learedi Price, 1934, Neospirorchis schistosomatoides Price, 1934, Monticelius indicum Mehra, 1939

Lepidochelys olivacea (ESCHSHOLTZ, 1829)

Trematoda (Anisakidae) - Sulcascaris sulcata (RUDOLPHI, 1819)

Trematoda (Pachypsolidae) - Pachypsolus irroratus (RUDOLPHI, 1819)

Trematoda (Pronocephalidae) - Pyelosomum renicapite (LEIDY, 1856)

Trematoda (Telorchiidae) - Orchidasma amphiorchis (BRAUN, 1899)

Fonte: Dados da pesquisa. Classificados de acordo com Ruggiero e colaboradores (2015).

A Tabela 1, a seguir, relaciona os helmintos parasitos de répteis e anfíbios no estado de São Paulo - Brasil e seu sítio de infecção no hospedeiro.

Tabela 1 - Relação das espécies de parasitas de anfíbios e répteis e seus sítios de infecção. BE - Bexiga Urinária; BO - Boca; CA - Cavidade Abdominal; CO - Cavidade Oral; ESO - Esôfago; EST Estômago; ID - Intestino Delgado; IG - Intestino Grosso; INT - Intestino; PU - Pulmão; RI - Rim; VB - Vesícula Biliar

\begin{tabular}{ccc}
\hline Grupo Parasitário & Espécie de Parasita & Sítio de infecção \\
\hline Acanthocephala & Acanthocephalus saopaulensis & CA, ID e IG \\
& Crepidobothrium gerrardii & ID e IG \\
\hline Cestoda & Cylindrotaenia americana & ID \\
& Oochoristicasp. & ID \\
Oonogetaeniasp. & ID \\
\hline Nematoda & Aplectanasp. & BE \\
& Brevimulticaecum sp. & BO e ESO \\
\hline & Brevimulticaecum pintoi & EST, ID e IG \\
& Cosmocerca brasiliensis & CA, EST, ID e IG \\
& Cosmocerca parva & EST e ID \\
& Cosmocerca podicipinus & ID e IG \\
& Cosmocercasp. & ID e IG
\end{tabular}




\begin{tabular}{|c|c|c|}
\hline Grupo Parasitário & Espécie de Parasita & Sítio de infecção \\
\hline & Cosmocercoidessp. & IG \\
\hline & Diaphanocephalus galeatus & INT \\
\hline & Falcaustra mascula & ID e IG \\
\hline & Hastospiculum digiticaudatum & CA \\
\hline & Hexametra boddaertii & CA e ID \\
\hline & Kathlania leptura & ID \\
\hline & Longibucca catesbeianae & EST \\
\hline & Ochoterenellasp. & CA, ID, IG, PU \\
\hline & Ortleppascaris alata & ID \\
\hline & Oswaldocruzia burseyi & EST, ID e IG \\
\hline & Oswaldocruzia fredi & EST e ID \\
\hline & Oswaldocruziasp. & ID e IG \\
\hline \multirow[t]{16}{*}{ Trematoda } & Oswaldocruzia subauricularis & EST, ID e IG \\
\hline & Oswaldofilariasp. & $\mathrm{CA}$ \\
\hline & Oxyascaris oxyascaris & ID e IG \\
\hline & Parapharyngodonsp. & ID e IG \\
\hline & Parapharyngodon largitor & EST e IG \\
\hline & Pharapharyngodon sceleratus & IG \\
\hline & Physalopteroides sp. & ID \\
\hline & Raillietnema simples & ID e IG \\
\hline & Rhabdias fuelleborni & PU \\
\hline & Rhabdias filicaudalis & PU \\
\hline & Schrankianasp. & ID e IG \\
\hline & Serpentirhabdiassp. & PU \\
\hline & Serpinema monospiculatus & ID \\
\hline & Skrjabinodonsp. & IN \\
\hline & Spiroxys figuereidoi & EST \\
\hline & Spiroxys sp. & EST e ID \\
\hline
\end{tabular}




\begin{tabular}{|c|c|c|}
\hline Grupo Parasitário & Espécie de Parasita & Sítio de infecção \\
\hline & Strongyloides ophidae & ID \\
\hline & Strongyluris oscari & EST e IG \\
\hline & Sulcascaris sulcata & IG \\
\hline & Thelandros sceleratus & CA e IN \\
\hline & Calycodes anthos & IG \\
\hline & Catadiscus propinquus & IG \\
\hline & Cheloniodiplostomum testudinis & ID e IG \\
\hline & Clinostomumsp. & CA \\
\hline & Cricocephalus albus & EST \\
\hline & Cricocephalus megastomum & EST \\
\hline & Deuterobaris proteus & ESO, ID e IG \\
\hline & Haematoloechus fuelleborni & PU \\
\hline & Haplometroides odhneri & $\mathrm{BO}$ \\
\hline & Infidum infidum & VB \\
\hline & Infidum similis & VB \\
\hline & Lophosicyadiplostomum sp. & $\mathrm{RI}$ \\
\hline & Mesocoelium monas & ID \\
\hline & Metacetabulum invaginatum & EST, ID e IG \\
\hline & Microscaphidium reticulare & ESO, ID e IG \\
\hline & Nematophila grandis & IG \\
\hline & Neoctangium travassosi & ESO, ID e IG \\
\hline & Ophisthogonimus sp. & CO e ESO \\
\hline & Orchidasma amphiorchis & EST e ID \\
\hline & Pachypsolus irroratus & ID \\
\hline & Pyelosomum renicapite & ID e IG \\
\hline & Physalopterasp. & EST \\
\hline & Pleurogonius longiusculus & ID e IG \\
\hline & Polyangium linguatula & ESO, ID e IG \\
\hline
\end{tabular}


Pronocephalus obliquus

ID e IG

Sticholecitha serpentis

CO e ESO

Travtrema stenocotyle

IG

Fonte: Dados da pesquisa.

\section{CONCLUSÃO}

Os resultados aqui exibidos mostram que, apesar do estado de São Paulo apresentar uma rica diversidade de répteis e anfíbios, são poucas as espécies relacionadas como hospedeiros para helmintos. A escassez de dados evidencia a necessidade de novos trabalhos que busquem gerar informações de campo, contribuindo, assim, para a compreensão das relações ecológicas que podem direcionar esforços voltados para a preservação do ecossistema. Além disso, o fato de a ordem Anura ter sido reportada como única hospedeira de helmintos no estado de São Paulo não é indicativo, necessariamente, de que não há outras ordens atuando como hospedeiros para diferentes helmintos.

Dentre os helmintos relacionados ao parasitismo de anfíbios e répteis, o filo Nematoda foi o mais comum, para ambos os grupos de vertebrados. Desses, muitos não puderam ser identificados pelos autores dos trabalhos, ou porque encontravam-se no estágio larval - o que dificulta a identificação - ou porque são necessárias mais pesquisas sobre a fauna helmintológica de animais silvestres, visando ampliar o conhecimento sobre o tema.

\section{REFERÊNCIAS}

\section{AGUIAR, A. Helmintofauna associada à Physalaemus cuvieri proveniente de duas áreas de Mata}

Atlântica. 2012. Trabalho de Conclusão de Curso - Instituto de Biociências, Universidade Estadual Paulista, Botucatu, SP, 2012.

AGUIAR, A. Helmintofauna associativa à anfíbios da Itha Anchieta, litoral norte do estado de São Paulo, Brasil. 2013. Dissertação (Mestrado) - Instituto de Biociências, Universidade Estadual Paulista “Júlio de Mesquita Filho", Botucatu, SP, 2013.

AGUIAR, A. et al. Amphibian Diseases. Herpetol Rev v. 45, n. 2, p. 13-17, 2014. 
AMATO, J. F. R.; AMATO, S. B. Técnicas gerais para coleta e preparação de helmintos endoparasitos de aves. Ornitologia e conservação: ciência aplicada, técnicas de pesquisa e levantamento. Rio de Janeiro: Technical Books, 2010. p. 369-393.

AMNH - American Museum of Natural History, New York, USA. Amphibian Species of the World: an Online Reference. Version 6.0. Electronic Database accessible at: http://research.amnh.org/ herpetology/amphibia/index.html. Accessed: 12 set. 2018.

ANDERSON, R. C. Nematode parasites of vertebrates: their development and Transmission. 2. ed. Wallingford - UK: CABI Publishing, 2000.650 p.

ANJOS, L. D. A. Ecologia de um lagarto exótico (Hemidactylus mabouia, Gekkonidae) vivendo na natureza (campo ruderal) em Valinhos, São Paulo. 2004. Dissertação (Mestrado) - Instituto de Biologia, Universidade Estadual de Campinas, Campinas, SP, 2004.

ANTONUCCI, A. M. Caracterização de agentes patogênicos virais e metazoários em rãs-touro, Lithobates catesbeianus, provenientes de ranários comerciais do Vale do Paraíba no estado de São Paulo, Brasil. 2009. Tese (Doutorado) - Instituto de Pesca, São Paulo, 2009.

ÁVILA, R. W.; SILVA, R. J. Checklist of helminths from lizards and amphisbaenians (Reptilia, Squamata) of South America. J Venom Anim Toxin, v. 16, n. 4, p. 543-572, 2010.

BAKER, M. R. Morphology and taxonomy of Rhabdias spp. (Nematoda: Rhabdiasidae) from reptiles and amphibians of southern Ontario. Can J Zool, v. 56, n. 10, p. 2127-2141, 1978.

BARRELLA, T. H. et al. Rhabdias filicaudalis n. sp. (Nematoda: Rhabdiasidae) from the snake Spilotes pullatus (Serpentes: Colubridae) in Brazil. J Helminthol, v. 84, n. 3, p. 292-296, 2010.

BLAUSTEIN, R. et al. Amphibian declines: judging stability, persistence, and susceptibility of populations to local and global extinctions. Conserv biol, v. 8, n. 1, p. 60-71, 1994.

CAMPOS, C. F. M. Fauna parasitária e alterações teciduais em três espécies de peixes dos rios Aquidauana e Miranda, Pantanal Sul Mato-grossense. 2006. Tese (Doutorado) Centro de Aquicultura Jaboticabal, Universidade Estadual Paulista “Júlio de Mesquita Filho", Botucatu, SP, 2006.

COSTA, H. C.; BÉRNILS, R. S. Répteis do Brasil e suas unidades federativas: lista de espécies. Herpetol Bras, v. 7, n. 1, p. 11-57, 2018. 
FORSTER, O. C. A influência parasitária de helmintos em aspectos reprodutivos de três espécies de anfíbios da RPPN Foz do Rio Aguapeí, município de Castilho, São Paulo, Brasil. 2017. Tese (Doutorado) - Instituto de Biociências, Universidade Estadual Paulista “Júlio de Mesquita Filho", Botucatu, SP, 2017.

FORTES, E. Parasitologia veterinária, São Paulo: Cone, 2004. p. 607.

JACOBSON, E. R. Parasites and parasitic diseases of reptiles. In: Infectious diseases and pathology of reptiles. CRC Press, 2007. p. 585-680.

LAFFERTY, K.D. Enviromental parasitology: What can parasites tell us about human impacts on the environment. Parasitol Today, v. 13, n. 7, p. 251-255, 1997.

MACHADO, A. C. R. et al. Helmintos parasitos em aves anseriformes que ocorrem em Goiás. Rev patol Trop, v. 35, n. 3, p. 185-198, 2006.

MADELAIRE, C. B. et al. Helminth parasites of Hypsiboas prasinus (Anura: Hylidae) from two Atlantic forest fragments, São Paulo State, Brazil. J Parasitol, v. 98, n. 3, p. 560-564, 2012.

MORETTI, E. H. Correlações comportamentais, fisiológicas e morfológicas da carga parasitária em Rhinella icterica (Anura: Bufonidae). 2011. Dissertação (Mestrado) - Instituto de Biociências, Universidade Estadual Paulista “Júlio de Mesquita Filho”, Botucatu, SP, 2011.

NOMURA, H. A. Q. Helmintofauna de duas espécies de anfíbios, Haddadus binotatus (Anura: Craugastoridae) e Ischnocnema guentheri (Anura: Brachycephalidae), da Mata Atlântica, no município de São Luiz do Paraitinga, São Paulo, Brasil. Trabalho de Conclusão de Curso - Instituto de Biociências, Universidade Estadual Paulista "Júlio de Mesquita Filho", Botucatu, SP, 2010.

NOVELLI, I. A. et al. First occurrence of Spirocamallanus sp. (Nematoda, Camallanoidea) in a freshwater turtle, Hydromedusa tectifera (Cope, 1869) (Testudines, Chelidae), from Brazil. Herpetol Notes, v. 7, p. 599-602, 2014.

PINHÃO, R. et al. Helminths of toad Rhinella icterica (Bufonidae), from the municipality of Botucatu, São Paulo State, Brazil. Neotrop Helminthol, v. 3, n. 1, p. 35-40, 2009.

PRICE, P. W. Evolutionary ecology of parasites. New Jersey: Princeton University Press, 1980.

QUIRINO, T. F. et al. New records of Helminths in Reptiles from five states of Brazil. Braz J Biol, v. 74, n. 4, 2018. 
RAILLIET, A. L'Emploi des Medicaments dans le Traitement des Maladies causées par des Nematodes. Tenth International Veterinary Congress, London, p. 733-749, 1915.

ROSSA-FERES, D. D. C. et al. Anfíbios do Estado de São Paulo, Brasil: conhecimento atual e perspectivas. Biota Neotrop, v. 11, p. 1-19, 2011.

ROSSELLINI, M. Caracterização da Helmintofauna de Helicops leopardinus (Serpentes, Colubridae) do Pantanal Sul, Mato Grosso do Sul. 2007. Dissertação (Mestrado) - Instituto de Biociências, Universidade Estadual Paulista “Júlio de Mesquita Filho”, Botucatu, SP, 2007.

RUGGIERO, M. A. et al. A higher level classification of all living organisms. PLoS ONE, v. 10, n. 4, p. e0119248, 2015.

RUNDQUIST, E. M. Reptile and amphibian parasites. Neptune: T. F.H. Publications, 1995. p. 64.

SANTOS, K. P. Implicações do parasitismo por nematódeos do gênero Rhabdias (Nematoda, Rhabdiasidae) em Crotalus durissus terrificus (Serpentes, Viperidae): alterações pulmonares, microbiológicas e hematológicas. 2005. Dissertação (Mestrado em Medicina Veterinária) Universidade Estadual Paulista “Júlio de Mesquita Filho”, Botucatu, SP, 2005.

SCHÜLLER, M. Pesquisa de protozoários e helmintos de interesse médico presentes nos excretas do pombo doméstico Columba livia domestica. 2004. 103 f. Dissertação (Mestrado) - Faculdade de Saúde Pública da USP, São Paulo, SP, 2004.

SCHWARTZ, H. O. Comunidades componentes de helmintos de anfíbios: análise comparativa entre duas localidades sob ação antrópica no município de São Luiz do Paraitinga, SP. 2010. Trabalho de Conclusão de Curso - Instituto de Biociências, Universidade Estadual Paulista "Júlio de Mesquita Filho", Botucatu, SP, 2010.

SEGALLA, M. V. et al. Brazilian amphibians: list of species. Herpetol Bras, v. 8, n. 1, p. 65-96, 2019.

SILVA, L. A. F. Helmintofauna associada a répteis provenientes da Reserva Particular do Patrimônio Natural Foz do Rio Aguapeí, Estado de São Paulo. 2014. Dissertação (Mestrado) - Instituto de Biociências, Universidade Estadual Paulista “Júlio de Mesquita Filho”, Botucatu, SP, 2014.

SILVA, R. J.; BARRELLA, T. H. Micrurus frontalis as a new host recorded for Haplometroides odhneri (Trematoda, Digenea, Plagiorchiidae). Rev Bras Parasitol Vet, v. 11, n. 1, p. 47-48, 2002.

SILVA, R. J. et al. Frequency of rhabdiasid nematodes in wild Crotalus durissus terrificus (Serpentes, Viperidae) from Botucatu region, São Paulo state, Brazil. J Venom Anim Toxin, v. 13, n. 1, p. 122-129, 2007. 
SMYTH, J. D. Introduction to animal parasitology. Cambridge University Press, 1994. p. 572.

STILES, C. W.; HASSALL, A. The Determination of Generic Types: And a List of Roundworm Genera, with Their Original and Type Species. US Department of Agriculture, Bureau of Animal Industry, 1905.

STUMPF, I. V. K. Ciclo evolutivo de Cylindrotaenia americana Jewell, 1916 (Cyclophyllidea: Nematotaeniidae) em Bufo ictericus Spix, 1824. Acta Biol Paranaense, Curitiba, v. 10, n. 11, p. 31-39, 1982.

STUNKARD, H. W. Life histories and systematics of parasitic worms. Syst Zool, v. 2, n. 1, p. 7-18, 1953.

TAVARES, L. E. R. et al. Helmintos endoparasitos de vertebrados silvestres em Mato Grosso do Sul, Brasil. Iheringia Série Zool, v. 107, 2017.

THATCHER, V. E. Patologia de peixes da Amazônia Brasileira, 1. Aspectos gerais. Acta Amazonica, Manaus, v. 11, n. 1, p. 125-140, 1981.

UETZ, P.; HOSEK, J. The reptile database. Accessible at: http://www.reptile-database.org. Accessed: Sep. 13, 2018.

VELOTA, R. A. M. V. Infecção por Helmintos em Thoropa taophora (Cycloramphidae) procedentes do Parque Estadual da Ilha Anchieta, Ubatuba, SP. 2012. Trabalho de Conclusão de Curso - Instituto de Biociências, Universidade Estadual Paulista “Júlio de Mesquita Filho”, Botucatu, SP, 2012.

VICENTE, J. J. et al. Description of Skrjabinodon spinosulus sp. n. (Nematoda, Oxyuroidea, Pharyngodonidae) from the Brazilian lizard Mabuya dorsivittata Cope, 1862 (Scincidae). Rev Bras Zool, v. 19, n. 1, p. 157-162, 2002.

VRCIBRADIC, D. et al. Helminth parasites of two sympatric lizards, Enyalius iheringii and E. perditus (Leiosauridae), from an Atlantic Rainforest area of southeastern Brazil. Acta Parasitol, v. 53, n. 2, p. 222-225, 2008.

VRCIBRADIC, D. et al. Nematodes infecting the skink Mabuya frenata in Valinhos, São Paulo State, southeastern Brazil. Amphibia-Reptilia, v. 20, n. 1, p. 333-339, 1999.

WERNECK, M. R. Estudo da helmintofauna de tartarugas marinhas procedentes da costa brasileira. 2011. Tese (Doutorado) - Instituto de Biociências, Universidade Estadual Paulista, Botucatu, SP, 2011. 
WERNECK, M. R. Helmintofauna de Chelonia mydas necropsiadas na base do projeto TamarIbama em Ubatuba, Estado de São Paulo, Brasil. 2007. Dissertação (Mestrado) - Instituto de Biociências, Universidade Estadual Paulista, Botucatu, SP, 2007.

WERNECK, M. R. et al. Gastrointestinal helminth parasites of Loggerhead turtle Caretta caretta Linnaeus 1758 (Testudines, Cheloniidae) in Brazil. PANAMJAS, v. 3, n. 3, p. 351-354, 2008.

WILSON, F. M. Levantamento da helmintofauna de Hypsiboas albomarginatus e Scinax hayii (Anura: Hylidae) da Ilha Anchieta, município de Ubatuba, SP. 2013. Trabalho de Conclusão de Curso - Instituto de Biociências, Universidade Estadual Paulista “Júlio de Mesquita Filho”, Botucatu, SP, 2013.

WILSON, F. M. Sazonalidade climática e os efeitos na helmintofauna parasita de Dendropsophus nanus (Anura: Hylidae) da RPPN Foz do rio Aguapeí, município de Castilho, São Paulo. 2015. Dissertação (Mestrado) - Instituto de Biociências, Universidade Estadual Paulista “Júlio de Mesquita Filho", Botucatu, SP, 2015.

YAMAGUTI, S. Rhabdias (Ophiorhabdias) horigutiin. subg., n. sp. (Nematoda) from the lung of a Japanese snake Natrix tigrina. Annot Zool Japon, v. 22, p. 8-10, 1943.

YAMAGUTI, S. Systema Helminthum - Cestodes. V. 2. London: Interscience Publishers, 1959. p. 860. 
1 Licenciada em Ciências Biológicas, Instituto Federal de São Paulo. E-mail: lesaninha@hotmail.com

2 Doutora em Ecologia de Ambientes Aquáticos Continentais; Professora EBTT, Instituto Federal de São Paulo. E-mail: gezasouza@ifsp.edu.br

3 Doutora em Anatomia dos Animais Domésticos e Silvestres; Professora EBTT, Instituto Federal de São Paulo.

E-mail: liviasantos@ifsp.edu.br

4 Doutora em Parasitologia, Professora EBTT, Laboratório de Zoologia, Curso de Licenciatura em Ciências Biológicas, Instituto Federal de São Paulo.

E-mail: tarsilaferraz@ifsp.edu.br

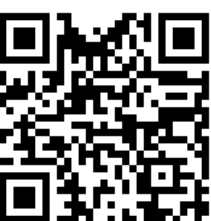

A autenticidade desse artigo pode ser conferida no site https://periodicos. set.edu.br

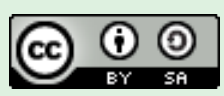

Este artigo é licenciado na modalidade acesso abertosob a Atribuição-Compartilhalgual CC BY-SA

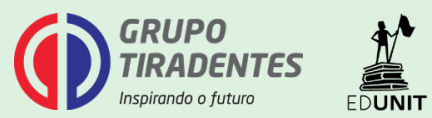

